\title{
A Solution for Cross-Docking Operations Planning, Scheduling and Coordination
}

\author{
Zhengping $\mathrm{Li}^{1}$, Cheng Hwee Sim ${ }^{2}$, Wei $\mathrm{He}^{1}$, Chong Chuan Chen ${ }^{2}$ \\ ${ }^{1}$ Singapore Institute of Manufacturing Technology, Singapore City, Singapore; ${ }^{2}$ Integrated Decision System Consultancy Pte Ltd., \\ Jalang Kilang Timor, Singapore. \\ Email: \{zpli,whe\}@simtech.a-star.edu.sg, \{chsim, ccchen\}@idsc.com.sg
}

Received July 29 $9^{\text {th }}$, 2011; revised November $11^{\text {th }}, 2011$; accepted December $15^{\text {th }}, 2011$

\begin{abstract}
Crossdocking is defined as an operational strategy that moves items through consolidation centers or cross docks without putting them into storage. As the need to move inventory faster increases, more logistics managers are turning to crossdocking but the ability to execute such strategy well depends on good planning, dynamic scheduling and coordination. This paper introduces our research and development work on cross docking solution in three aspects: optimized planning on container grouping, clustering, sequencing and allocating containers to docks; real-time scheduling handles the dynamics of container arrivals and actual pallet transfers; and cross-docking coordination conducts real-time task assignment/sequencing and resource management to deal with dynamic changes.
\end{abstract}

Keywords: Logistics; Crossdocking; Planning; Optimization

\section{Introduction}

Crossdocking is a practice in logistics of unloading materials from incoming trailers and loading these materials in outbound trailers, with little or no storage in between [1]. It is also defined as an operational strategy that moves items through flow consolidation centers or cross docks without putting them into storage [2]. With the Just-in-time concept being accepted widely, the implementation of crossdock operations repositions the focus from warehousing inventory to the one of managing inventory through-flow in transit from suppliers to customers. Crossdocking becomes an important strategy to improve the performances of supply chain services in movement velocity, inventory and quick repsonse.

Customers are demanding speed and accuracy, and warehouses are undergoing transformation-from a solid physical facility used to house "mistakes" in forecasting called inventory to one that distributes products in the shortest possible time at the lowest possible cost. Growing number of partners and delivery points, shrinking volume but rising frequency of orders, ever shorter delivery time and stricter working hours of drivers, and ever increasing pressure to reduce inventories all contribute to growing pressures on supply chains. A trend toward smaller and fewer warehouses may well translate into more crossdocking operations in the 21st century [3].

In cross-docking, shipments typically spend less than
24 hours in a crossdock. The purpose of crossdocking include reducing inventory cost, increasing inventory turns, consolidating transportation, increasing throughput, and reducing operation costs associated by eliminating unnecessary handling and storage. It offers a way to increase inventory velocity. However, systems to optimize crossdocking had so far mainly focused on finding the best location to cross-dock, the best shape of cross docking platforms, and how best to batch the arrival of the trucks or containers (container in this document refers to both containers as well as trucks). The actual crossdocking operations are still managed with manual rules that give sub-optimal results. This paper outlines a prototyping system developed to optimally plan the arriving of containers, the allocation of containers to docks, and the sequencing of tasks for forklifts to maximize throughput. We also consider the handling of dynamic uncertainties encountered in execution. The research and development work was supported by Integrated Decision System Consultacy Pte Ltd and a T-UP grant from A*Star (Agency for Science, Technology and Research, Singapore). It was originally meant to address a military need to quickly crossdock ammunition containers stored underground during mobilization.

\section{Literature Review}

Research work on crossdocking has been mainly conducted on such areas as crossdocking system and layout 
design, network design, and crossdocking operations planning and scheduling.

Rohrer [4] discussed modeling methods and issues as they are applied to crossdocking systems. He described how simulation helps to ensure success in crossdocking system design by determining optimal hardware configuration and software control. Apt and Viswanathan [5] addressed a framework for understanding and designing crossdocking systems and discussed techniques that can improve the overall efficiencies of logistics and distribution networks. Napolitano [6] classified the various types of crossdocking operations. These include manufacturing, distributor, transportation, retail and opportunistic crossdocking. Napolitano also describes crossdocking as the "JIT in the distribution arena". Manufacturing crossdocking constitutes the receiving and consolidating of inbound supplies where a manufacturer can use a warehouse to receive supplies of parts for meeting demands ascertained from an MRP. In retail crossdocking, retailers receive products from multiple vendors who use distributors with multiple warehouses. In general, crossdocks are complex, requiring a high degree of coordination between suppliers, customers and distributors to create shipments based on anticipated supplies and demands [7].

Donaldson et al. [8] studied a network of crossdocks for the US Postal Service where 148 Area Distribution Centers serve as crossdocks, each receiving, sorting, packing and dispatching mail according to operating schedules. Each distribution center serves as an origin as well as destination node where schedules were driven by mail delivery standards. Ratliff et al. [9] studied a loaddriven network, in which deliveries take place when there are sufficient products waiting for transportation. They studied the North American automobile delivery systems to determine the ideal number and location of crossdocks in a network and how shipments flowed between them. In their study, a minimum inventory strategy was the key in attempting to minimize the number of vehicles at the mixing center (crossdocks). Chen, Guo and Lim [10] studied crossdocking network scheduling where time windows for deliveries and pickups are considered. They also considered crossdock-handling costs which are use to penalize delays.

Also there are quite amount of research work on crossdocking facility design. Barthold and Gue [11] determined the best shape for a crossdock by analyzing the assignment of receiving and shipping docks. The staging of products in a crossdock to avoid floor congestion and increase throughput has also been studied together with the effects of different combinations of number of workers in receiving and shipping on throughput [12]. A simulated annealing procedure was used to construct effective layout to reduce labour costs [13]. A simulation model of a generic cross-docking facility [14] to examine the operational risks associated with individual crossdocking facilities within a company's distribution network under a dynamic environment.

In all crossdocking situations, the timing of delivery and pickup is crucial to effective operations. The performance of crossdocking depends on good planning and scheduling. Li et al. [15] considered short term scheduling of material handling inside the terminal for a given truck schedule. They model the jobs and resources as a machine scheduling problem and present a meta-heuristic for its solution. Chen, Guo and Lim [10] studied crossdocking scheduling where time windows for deliveries and pickups are considered. They also considered crossdock-handling costs which are use to penalize delays. Yu and Egbelu [16] studied the scheduling issue of inbound and outbound trucks in crossdocking systems with temporary storage. They try to find the scheduling sequence for both inbound and outbound trucks to minimize total operation time when a storage buffer to hold items temporarily is located at the shipping stock. Chen and Lee [17] develop polynomial approximation algorithm and branch-and-bound algorithm to minimize the makespan for products going through a crossdocking facility. McWilliams et al. [18] covered a specific truck scheduling problem at a parcel hub. A simulation-based scheduling approach with an embedded genetic algorithm is proposed.

Based on our reviews, crossdocking planning and scheduling problem is still not properly investigated. Although some research proposed models on truck scheduling and dock assignment problems related to crossdocking, most of the studies are in the research stage, especially the time for getting the solution is still too long and un-feasible to real scenarios. Also most of the solutions do not consider handling of uncertainties in the scheduling and coordination of crossdocking operations. There is still shortage of effective operations planning solution for crossdocking with considerations of vehicle arriving, container allocation, and exchanging sequencing. There is also shortage of real-time scheduling for handling dynamics on container arriving, allocation and resource uncertainties. These issues are discussed in this paper.

\section{Research Issues}

As discussed previously, operational performance in crossdocking greatly depends on good planning, scheduling and coordination. We studied technologies and algorithms to provide optimized solution for cross-docking planning, scheduling and operations coordination:

- Optimized planning for container grouping, clustering, sequencing and allocation with build-in mathematic algorithm.

- Real-time scheduling handles the dynamics of con- 
tainer arrivals and actual cross-docking operations.

- Cross-docking coordination conducts real-time task generation, sequencing and assignment and resource allocation.

We consider a pure cross-docking pre-allocation scenario. The information such as what cargo should be delivered to where in a particular day (information come from ERP, Order management systems), facilities (size, shape), and resources (forklifts, doors) are taken as input. Our research focuses on the following crossdocking operation issues:

- When should a vehicle arrive in the cross-dock?

- Which group of containers should enter the crossdock with time-overlapping?

- Which dock should a container be allocated to?

- What should be the sequence of exchanging of pallets between containers?

- How to handle dynamics on arriving, processing, and facility uncertainty?

- How to produce the tasks which would be assigned to forklifts for exchanging pallets?

A number of assumptions are applied in the crossdocking model:

- A pure crossdocking scenario is considered where pallets are stored in containers and are exchanged between containers.

- Product assumptions: multiple types of products are processed in the crossdocking center and the products in the incoming containers are known. Products are packaged in pallets. One pallet includes only one type of product.

- Containers assumptions: a container can take multiple pallets; each container has a capacity limitation.

- Pallets assumptions: the pallets have standard dimensions and volume. The transfer cost of pallets would be the same if the moving distance is the same. The transfer cost of a pallet is independent of the number of pallets being transferred.

- Crossdock facility: a crossdock has a number of doors where containers can be allocated for exchanging pallets. A door can be used for both inbound and outbound purposes. Forklifts are used in crossdock for pallet unloading, movement, and loading.

\section{Design and Development Solution}

To answer the above raised questions, we developed a crossdocking solution which solves the problems in three layers (as shown in Figure 1): Planning layer, scheduling layer, and coordination layer.

The planning layer produces the groups and clusters of containers based on their exchanging relationships. It also determine the time for a container to arrive the rossdock and the allocation plan to assign containers to

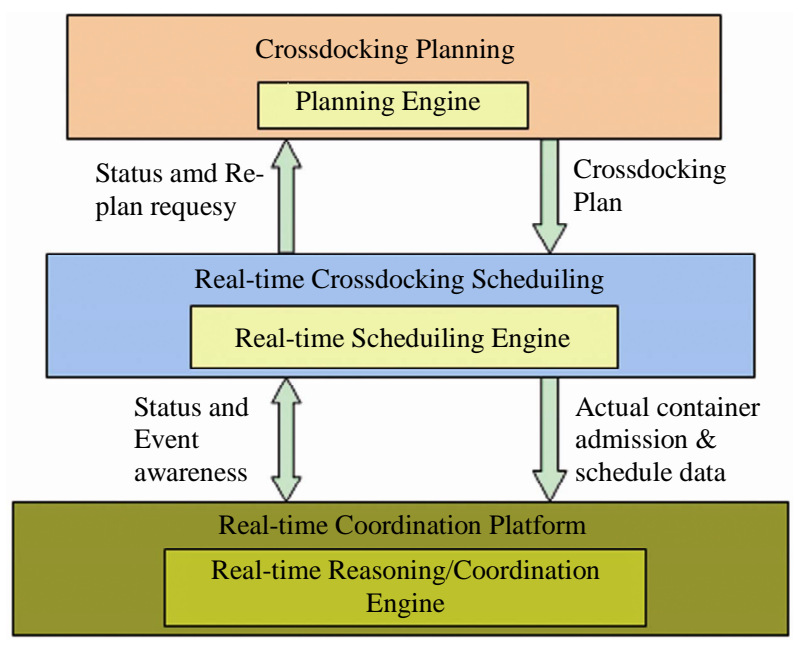

Figure 1. Crossdocking solution framework.

docks so that the containers can complete the crossdocking operations between each other. The scheduling layer produces more detail operations schedule and task list. It also handles dynamics in container arriving late, container processing late and resource uncertainties in the crossdock. The coordination layer is for real-time operations coordination and execution control, mainly is to control the resources (such as forklifts) to execute the tasks and complete the exchanges of pallets.

Graph theory is applied in the design of algorithm of crossdocking planning/scheduling. We mapped pallet exchange problem between containers as a graph theory problem. A container is modeled as a vertex and pallets exchanged between the containers are modeled as edges. So the crossdocking operations problem can be mapped to a connected weighted di-graph G.

The design and development of these layers are introduced in more detail in the following sections.

\subsection{Crossdocking Planning}

The planning solution is designed to produce optimized cross-docking plan considering minimize the total processing time of a set of containers. Figure 2 shows a planning result with the most left column and the top row represents containers. The mid part of the matrix represents the number of pallets to be exchanged between any two containers. It shows that the system can identify groups, clusters, and sequence the groups, clusters and containers.

Crossdocking planning includes the following aspects of design and optimizations: grouping, clustering, container sequencing, and allocation of containers to docks.

- Grouping: Identification of the groups of containers that have pallet exchange relationships between them. A group includes those containers which are linked directly or indirectly by the exchanges (edges). A 
group is represented by the yellow color in Figure 2.

- Clustering: Identification of the containers (vertices) with strong connectivity relationships within the same group. By this, the appropriate sequence of clusters of containers to enter the crossdocking area could be determined since a cluster with outputs to other clusters should be allocated earlier. A cluster is represented in green color in Figure 2.

- Identify minimum door requirements for groups (clusters): A crossdock needs to cater for multiple customers, so multiple group of containers compete for limited door resources. This function is to calculate the minimum number of docks needed is for completing the exchange of a group without re-admission of containers to the crossdock.

- Optimized allocation of groups and containers to docks: this is to determine which container should be assigned to which door with consideration of minimizing movement distance in pallet exchanges (Figure 3).

Facility data, resource data, and pallet transferring requirements are defined in Excel sheet as input to the planning model. An algorithm is also designed to identify the minimum door requirements for groups and clusters which are very useful in the assignment of containers to

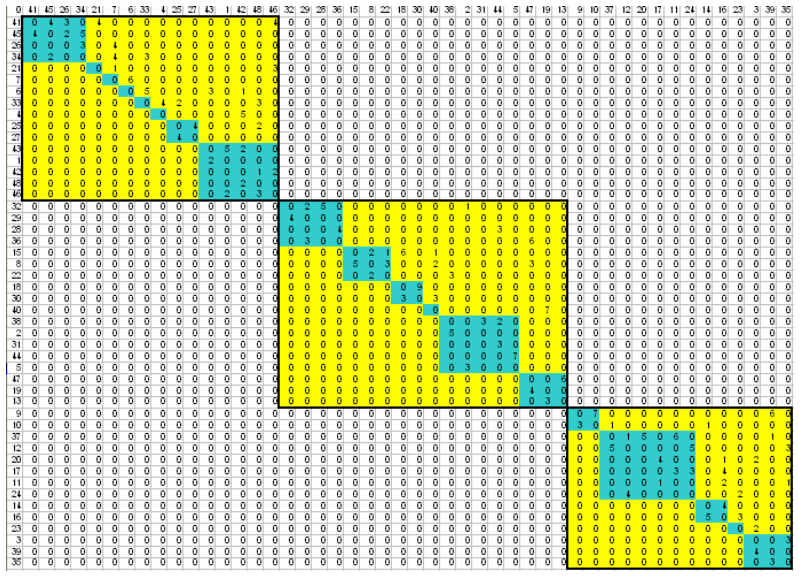

Figure 2. Crossdocking plan.

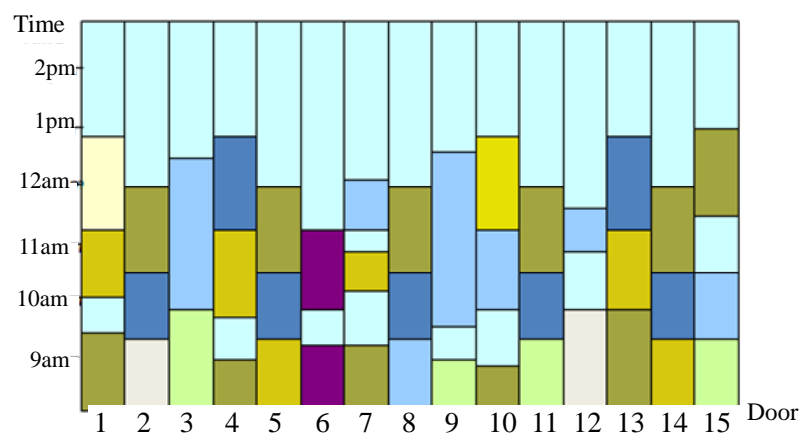

Figure 3. Container assignment to doors. doors especially when door becomes a critical resources.

Currently, most crossdocks rely on supervisors to manually create docking assignments, or use WMS or TMS solutions to schedule appointments directly to specific docking bays by time block. These simplistic approaches often result in sub-optimal sorting assignments due to their inability to account for the complexity of sorting operations, especially during peak flows. In addition, they cannot accommodate dynamic changes that are inevitable in execution, leading to inefficient dock utilization and pallet transfers.

Unlike manual or other less capable appointment systems, our cross-docking planning and scheduling engines increase sorting capacity by employing advanced modeling to consider container relationships, sorting resources, and facility configuration. Container groups are allocated to the right areas considering priorities. Bin-packing algorithms are used for optimal container allocation (Figure 3). Bins (refer to doors) are occupied in the time dimension with the right height by the right container to make exchanging of pallets possible. Containers with high exchanging relationship are allocated to neighbor docks to reduce exchanging cost and time. The plan ensures that containers are admitted to cross-dock and pallets are transferred in a smooth and continuous way.

\subsection{Real-Time Crossdocking Scheduling and Admission Control}

Uncertainty is common in crossdocking systems. Trucks may not arrive in time, container processing could be late or earlier than planned, and resources could be out of operations etc. In crossdocking, containers (trucks) will be admitted to a sorting area dynamically based on actual arrivals and progress of crossdocking operations. A crossdocking system must adapt to dynamic changes, otherwise crossdocking cannot be realized effectively.

We design the logics of the scheduling layer which takes planning result as input. When dynamic changes are detected, re-scheduling module will be triggered to produce a more feasible and better schedule. The current developed scheduling solution includes the logic to make decision on how to handle the dynamics in the following situations:

- Cross-docking operation processing doesn't follow existing plan, e.g., container processing late.

- Containers arrive later than the plan.

- Resource out of service.

If the processing of a container is later than planned or a container arrives later, the rescheduling can determine how this will affect other container processing and what action should be taken. In the container arriving late situation, depends on the time delayed, the system can make the decision on if the related containers should wait 
in the crossdock, in the outside waiting area, or just leave without get the input from the delayed container. The system can redefine new groups (for the later container and the waiting container) and arrange time slot and docks for the new groups, or define virtual container to be processed in the next day for the container which leave without taking the inputs from the delayed containers.

The rescheduling module also includes the functions of grouping, clustering and sequencing and dock allocation which are similar with the planning module. Crossdocking scheduling and dynamic container admission will combat peak and valley waves by balancing demand and resource availability and therefore smooth the workload throughout based on the service availability of cross-dock dock and forklift.

The admission control module is in-between of planning layer and coordination layer, acts as the role for real-time container admission to crossdock by taking schedule and real-time information from coordination layer as inputs, and generating real-time container admission plan based on input information. Admission logics are designed in such a way that the admission is conducted based on existing schedule when there is a container leaving the crossdock upon finishing its pallet exchanging. However, re-scheduling will be performed if container processing is very late or very earlier, dock is broken-down, or container arriving is late etc. In the case of re-scheduling, containers will be admitted into crossdock based on the new schedule.

\subsection{Coordination Platform Design and Development}

The purpose of crossdocking coordination is to maximize throughput and improve operations efficiency of crossdocking operations with resource constraints. A smooth sorting workload ensures that the right resources are available to avoid bottlenecks and maximize throughput.

A cross-docking master scenario and related assumptions have been defined in the coordination layer. Processes for the handling of general containers and pallets have been modeled and confirmed. Task assignment and resource scheduling at the operational level match tasks with resources. Task assignment logics and processes are designed into the system according to user requirements defined. In addition, specifications for resources (containers, cross-docking docks, forklifts, and staging areas) and related control logics are included in the coordination module. Locations and distance norms are also defined for container and forklift allocation purposes. Time norms are defined for task control and assignment. GUI requirements for different application modules have also been developed.
There are three main modules in the coordination layer. They are business application module, foundation module (workflow and database) and GUI module. The GUI module is for user's interaction with the system including Main GUI for Control, Scenario Definition, Progress Monitoring and Control, Task Management, Resource Management, Norms Management. The business application module provides core business logics for process execution and support such as assignment implementation, process implementation, mode control for operational/auto-run, resource (forklift, dock \& staging area) controller, main application controller, and assignment management (resource allocation, assignment and task fulfillment). Foundation module provides system level services to support fundamental object and information management, database access and interface with workflow engine.

The coordination layer can be run standalone as well as integrated with the scheduling layer. To run as a standalone system, the data of containers and pallets can be loaded from an excel file and the information can be used to create internal objects. For integrated running of the system, the containers will be admitted by admission module in real-time based on the schedule that is created dynamically according to real-time situation. In this way, coordination layer is able to handle continuous container admission and was able to manipulate data in a real-time manner. Information of containers and its pallets that are not admitted yet will be only used for planning and estimation purpose in coordination.

An event-based integration framework has been developed for integrating the planning, scheduling and coordination layers. It is a server-client integration mechanism which can bring the advantages to the 3-layer crossdocking system, such as portable and flexible configuration and plug \& play. An event server can has several event clients registering with it. Information can be transferred among the clients when events are triggered. Data interface format has been defined in order for the 3 layer to communicate and transfer data based on the same protocol. It includes data interface format defined for container-processing completion event, container arrives late event, dock service status change event, and container admission event. Event management mechanism has been implemented in the coordination layer and admission layer to apply the logics for events emitting, receiving and handling.

\section{Solution Validation and Advantages}

\subsection{Prototype Testing}

The input data for the prototype include container data, container pallet exchange information, facility data (include distance between doors), and time norms informa- 
tion. The container data represents the containers that will go through the crossdock in a particular time period; container pallet exchange information is about what pallets would be exchanged between containers; facility location information include the relative distance between locations such as docks and staging area; and the time norms information represents the time needed for a standard loading, unloading, or movement operations.

Input data for prototype testing and has been designed. We have debugged and tested the prototype system with predefined input data for both different scenarios. The simple scenario includes 7 pallets from 8 containers while the complex scenario is to test with the near real case for 390 pallets exchanging for 48 containers. Results obtained from test runs show the system works by following expected logics and meet the targets.

We have tested the planning and scheduling modules independently with multiple sets of data. We also have done integration testing of the planning and scheduling modules. The testing shows the communication between scheduling and coordination are effective. The planning and scheduling result can be used to trigger operation and the status information could be feedback to rescheduling and it can trigger rescheduling if it is necessary according to the logic included.

\subsection{Solution Advantages}

Logistics companies in many developing countries, especially in places like China, plan manually and are thus forced to organize themselves in simplified ways to handle real world complexities. They therefore suffer from lack of scale and know-how to exploit technology to overcome this problem. The cross-docking development in IDSC provides optimized solution for crossdocking planning, scheduling and operation coordination.

The developed solution has been presented to Singapore local and MNC logistics companies. They commented that the solution was quite impressive and expressed high interest on the application of the solution. Discussion of potential application project is still in progress. We also proposed to a China logistics company which has the requirement to speed up their forwarding business for such customers as Wal-Mart. Since many countries around the world share the same challenges, the projected market size and sales for the technologies and associated business model would be quite big. Another major potential for optimal cross-docking planning and real-time coordination is large-scale real-time military logistics systems. The US military is leading the push in this area but Singapore's growing sophistication in ammunition logistics may also provide a boost.

The solution aims to improve cross-docking operations not only with higher speed and lower cost, but also copes well with dynamic changes. The advantages of the solution include:

- Maximize throughput and improve efficiency through optimization and coordination such that containers arrive at the right dock at the right time and pallets are transferred in the right sequence even in the face of dynamics.

- Sorting based on optimized container allocation: unlike traditional systems, our cross-docking engines increase sorting capacity by employing advanced modelling to consider container relationships and facility configuration. Containers are admitted to crossdock and pallets are transferred in a smooth and continuous way.

- Lower inventory cost and improved service levels: updating order quantities at the crossdock so that discrepancies between what was ordered and what is really needed could be reduced. This effectively reduces the safety stock, and also raises the service levels.

- Reduce storage and material handling cost: Crossdocking smoothly and speedily will not only lower material handling costs but also contribute to lower cost in the supply chain as storage is bypassed and velocity is increased.

- Consideration for dynamic effects and item priority: dynamic scheduling and admission will combat peak and valley waves by balancing demand and resource availability.

The developed solution has the following benefits to users:

- Suppliers and manufacturers would know exactly when the cargo should be sent to cross-docking center, and when the needed material would arrive. This will avoid sending vehicle too early or too late.

- Distribution and crossdocking center will enjoy easier and smoother crossdock operations with faster optimized planning and scheduling that maximizes the throughput of these facilities.

- Transportation service providers will see better plans that coordinate their vehicle arrival times more accurately and get their vehicles on the road again faster.

- Recipients of the goods enjoy reduced inventory and improved customer service levels as their inventory can be replenished more frequently and stock-outs are averted with higher velocity.

\section{Conclusions}

This paper introduces a solution developed for realistic crossdocking operations. It includes three layers of modules: planning layer for container arriving, grouping and allocation; scheduling layer for dynamic event handling; and coordination layer for execution control and resource 
management.

The solution developed can be used to produce a clear plan for continuous container admission and processing operations quickly. So it enables the crossdock manager to response to changes faster and avoids chaos in crossdocking operations which are with high requirements on timing and low inventory. It also help maximizing throughput and improving facility efficiency through optimization and coordination such that containers arrive at the right dock at the right time and pallets are transferred in the right sequences even in the face of dynamics. Inventory cost could also be reduced for the crossdock operators and also for end customers due to the optimized sorting and improved responding speed. Several potential users in Asia have expressed interests to the solution and one of them starts the implementation of this solution since March 2007.

Future work will be conducted in the areas of crossdocking operations planning and scheduling for the combining scenario of crossdocking and warehousing; product mixing and matching for crossdocking to meet customer demand dynamically.

\section{REFERENCES}

[1] K. R. Gue, "Crossdocking-Just-in-Time for Distribution, Graduate School of Business \& Public Policy,” Naval Postgraduate School, Monterey, 2001.

[2] T. Babics, "Crossdocking in the Sales Supply Chain: Integration of Information and Communication Relationships," Periodica Polytechnica Transportation Engineering, Vol. 33, No. 1-2, 2005, pp. 69-76.

[3] B. Thompson, "21 Warehousing Trends in the 21st Century, IIE Solutions,” 1999. http://solution.iienet.org

[4] M. Rohrer, "Simulation and Crossdocking," Proceedings of the 1995 Winter Simulation Conference, Arlington, 3-6 December 1995, pp. 846-849. doi:10.1109/WSC.1995.478868

[5] U. M. Apte and S. Viswanathan, "Effective Crossdocking for Improving Distribution Efficiencies,” International Journal of Logistics: Research and Applications, Vol. 3, No. 3, 2000, pp. 291-302. doi:10.1080/713682769

[6] M. Napolitano, "Making the Move to Crossdocking: A Practical Guide to Planning, Designing, and Implementing a Cross Dock Operation,” Warehousing Education and Research Council, Oak Brook, 2000.
[7] B. Shaffer, "Implementing the Crossdocking Operation," IIE Solutions, Vol. 30, No. 5, 2000, pp. 20-23.

[8] H. Donaldson, E. L. Johnson, H. D. Ratliff and M. Zhang, "Network Design for Schedule-Driven Cross-Docking Systems,” Georgia Tech TLI Report, 1998.

[9] H. D. Ratliff, J. V. Vate and M. Zhang "Network Design for Load-Driven Cross-Docking Systems, TLI Webpage," 2001. http://www.isye.gatech. edu/research/files/misc9914.pdf

[10] P. Chen, Y. Guo, A. Lim and B. Rodrigues, "Multiple Crossdocks with Inventory and Time Windows," Computers and Operations Research, Vol. 33, No. 1, 2006, pp. 43-63. doi:10.1016/j.cor.2004.06.002

[11] J. J. Bartholdi III and K. R. Gue, "The Best Shape for a Crossdock,” Transportation Science, Vol. 38, No. 2, 2000. doi:10.1287/trsc.1030.0077

[12] J. J. Bartholdi, K. R. Gue and K. Kang, "Staging Freight in a Crossdock," Proceedings of the International Conference on Industrial Engineering and Production Management, Quebec City, 20-23 August 2001.

[13] J. J. Bartholdi III and K. R. Gue, "Reducing Labor Costs in an LTL Crossdocking Terminal," Operations Research; Vol. 48, No. 6, 2002, pp. 823-832. doi:10.1287/opre.48.6.823.12397

[14] G. M. Magableh, M. D. Rossetti and S. Mason, "Modeling and Analysis of a Generic Crossdocking Facility," Proceedings of the 2005 Winter Simulation Conference, Orlando, 4-7 December 2005, pp. 1613-1620.

[15] Y. Li, A. Lim and B. Rodrigues, "Crossdocking-JIT Scheduling with Time Windows,” Journal of the Operational Research Society, Vol. 55, 2004, pp. 1342-1351. doi:10.1057/palgrave.jors.2601812

[16] W. Yu and P. J. Egbelu, "Scheduling of Inbound and Outbound Trucks in Crossdocking Systems with Temporary Storage," Europe Journal of Operations Research, Vol. 184, No. 1, 2007, pp. 377-396. doi:10.1016/j.ejor.2006.10.047

[17] F. Chen and C. Y. Lee, "Minimizing the Makespan in a Two-Machine Cross-Docking Flow Shop Problem,” Europe Journal of Operational Research, Vol. 193, No. 1, 2007, pp. 59-72. doi:10.1016/j.ejor.2007.10.051

[18] D. J. McWilliams, P. M. Stanfield and C. D. Geiger, “Minimizing the Completion Time of the Transfer Operations in a Central Parcel Consolidation Terminal with UnequalBatch-Size Inbound Trailers," Computers \& Industrial Engineering, Vol. 54, No. 4, 2008, pp. 709-720. doi:10.1016/j.cie.2007.10.006 